\title{
Stress Echocardiography: Concept and Criteria, Structure and Steps, Obstacles and Outcomes, Focused Update and Review
}

\author{
Antoine Kossaify ${ }^{\mathrm{a}, \mathrm{b}, \mathrm{c}}$, Elie Bassi1 ${ }^{\mathrm{a}, \mathrm{b}}$, Mikhael Kossaify ${ }^{\mathrm{a}, \mathrm{b}}$
}

\begin{abstract}
Stress echocardiography (SEC) is a technique established more than 35 years ago; however, it is still poorly implemented in many countries and institutions, and this reluctance may be related to many obstacles such as operator skills, lack of awareness or institutional policy. Stress echo was initially used for assessing coronary artery disease (CAD), with respect to myocardial viability, using wall motion response; however, current use of stress echo extends beyond CAD, such as valvular heart disease and diastolic stress test. Dobutamine is a commonly used agent when pharmaceutical approach is implemented. With regard to $\mathrm{CAD}$, there are four stress responses: normal, ischemic, viable and necrotic. A low dose dobutamine protocol is recommended in patients with baseline wall motion abnormalities, and a very low dose dobutamine is used in low flow low gradient aortic stenosis in order to check the flow and contractility reserve. Of note, respecting protocols, indications and contraindications are important to avoid or minimize risks of complications during the procedure. This article presents a focused update and review regarding SEC, along with an overview of the different indications, structures and steps, and obstacles and outcomes; also the article aims to highlight more awareness and sensitization on this useful technique.
\end{abstract}

Keywords: Echocardiography; Stress; Echo; Dobutamine; Indications

\section{Introduction}

Stress echocardiography (SEC) was initially developed in 1979 for evaluation of patients with known or suspected cor-

Manuscript submitted February 26, 2019, accepted January 28, 2020

${ }^{a}$ Cardiology Division, Echocardiology Unit, University Hospital Notre Dame des Secours, PO Box 3, Byblos, Lebanon

${ }^{b}$ Holy Spirit University of Kaslik (USEK), School of Medicine, St Charbel Street, Byblos, Lebanon

${ }^{\mathrm{c} C o r r e s p o n d i n g ~ A u t h o r: ~ A n t o i n e ~ K o s s a i f y, ~ C a r d i o l o g y ~ D i v i s i o n, ~ U n i v e r s i t y ~}$ Hospital Notre Dame des Secours, PO Box 3, Byblos, Lebanon.

Email: antoinekossaify@yahoo.com

doi: https://doi.org/10.14740/cr851 onary artery disease (CAD); however, this technique is still poorly implemented in many countries and facilities [1]. The classical stress test known as electrocardiography stress test is usually performed to diagnose $\mathrm{CAD}$; however, a significant proportion of patients are unable to exercise adequately because of advanced aging, physical limitations and limiting comorbidities [2].

In this regard, SEC allows performing a cardiac stress exam without physical stress (pharmacological stress testing), and the main indication is known or suspected CAD; moreover, SEC provides information that an electrocardiography stress test does not offer, such as diagnosis myocardial viability, severity and prognosis of CAD and of many cardiac conditions beyond CAD, such as valvular heart diseases $[2,3]$.

\section{Concept and Criteria}

\section{Concept}

During SEC, stress is commonly applied to the cardiovascular system pharmacologically, either with dobutamine, dipyridamole, or adenosine, given that physical exercise often induces motion artifacts. Dobutamine stress echocardiography (DSE) is the most commonly used agent given that dobutamine is more convenient, also it yields higher sensitivity and its side effects are relatively controllable compared to other products [4].

\section{Criteria to perform the test (indications)}

SEC is most commonly used in patients with known or suspected CAD, namely when exercise electrocardiography stress test is either non-feasible, non-diagnostic or non-interpretable (e.g. locomotor insufficiency, ambiguous results, pacemaker, bundle branch block, significant electrocardiographical changes at rest making interpretation during stress difficult, etc.) [4, 5].

The following list presents the main indications of SEC [35]: 1) CAD: diagnostic and prognostic (i.e. including preoperative risk assessment, search for ischemia location, myocardial viability);2) Evaluation of prognosis and severity of valvular heart disease; 3) Diastolic stress echocardiography; 4) Diagnosis of microvascular CAD; 5) Evaluation of non-ischemic 

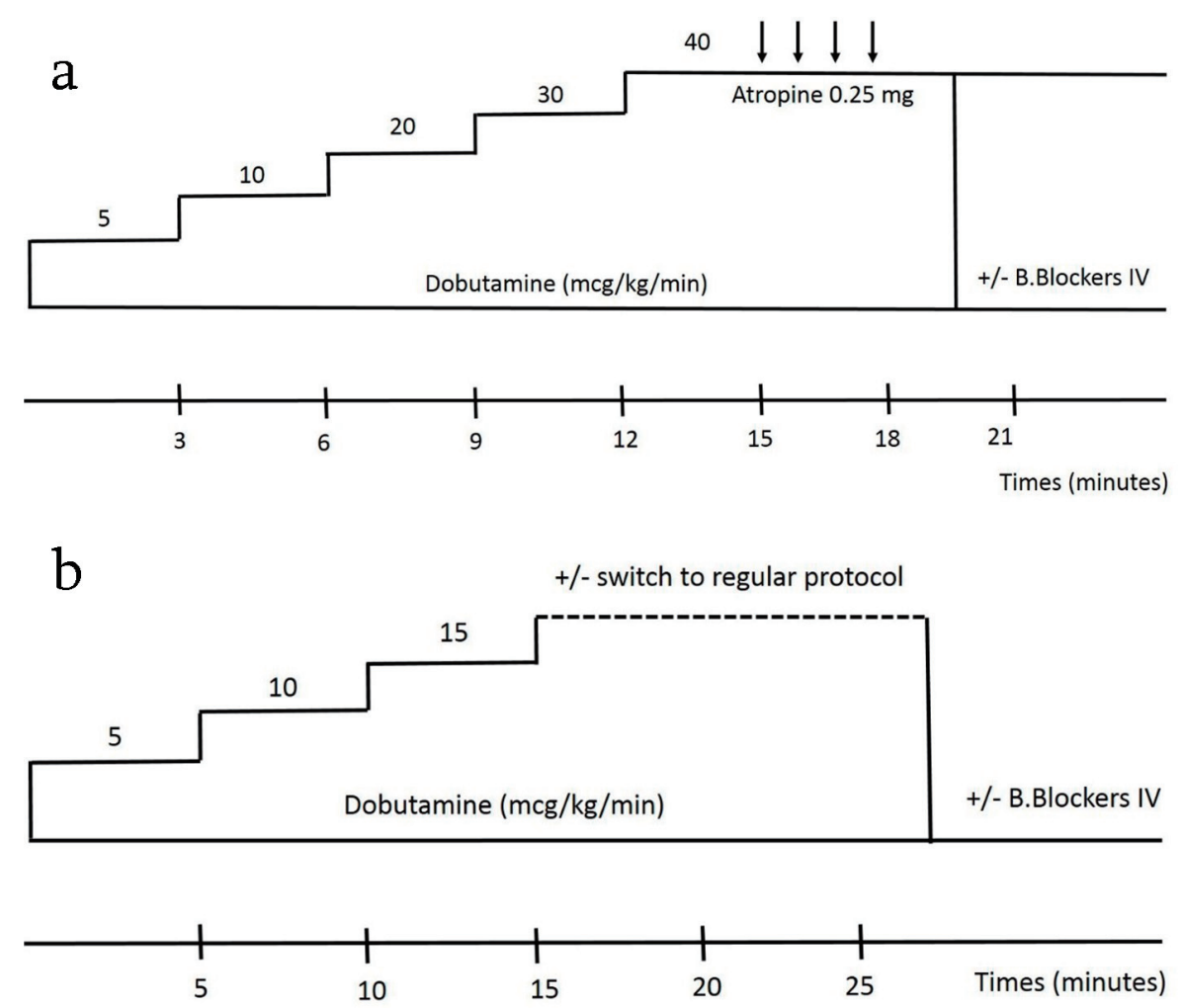

Figure 1. Image showing regular DSE protocol (a) and (b) low dose DSE protocol. DSE: dobutamine stress echocardiography.

dilated cardiomyopathy and hypertrophic cardiomyopathy; 6) Evaluation of pulmonary hypertension; 7) Evaluation for some patients with suspicious cardiac etiology of exertional dyspnea; 8) Evaluation of some patients with congenital heart disease; 9) Evaluation of some patients after heart transplantation; 10) Evaluation of athletes and subjects submitted to extreme physiology (e.g. diving, high altitude).

\section{Structure and Steps}

\section{Structure of the technique}

SEC is implemented using either pharmacological approach or physical stress; however, pharmacological approach is the most commonly used technique given that such an approach helps to avoid the challenges caused by exercise echocardiography such as excessive chest wall motion, allowing acquiring better image quality. A baseline echocardiogram should be obtained at rest. Moreover, images are examined at rest to assess the quality of sonographic signal and the potential presence of technical limitations $[6,7]$. DSE is accomplished by administering incremental dosage of dobutamine infusion. The patient must have an adequate venous access, and also he (or she) is attached to a cardiac monitor in order to observe heart rate, rhythm and blood pressure [6].

Dobutamine infusion is started at a dose of 5 or $10 \mu \mathrm{g} / \mathrm{kg} /$ min increasing gradually to 20,30 and finally to $40 \mu \mathrm{g} / \mathrm{kg} / \mathrm{min}$ with an interval of 3 min for each dosage. The test can be halted when $85 \%$ of the age-predicted heart rate is reached, and also the test is halted when significant symptoms or intolerance signs develop, namely when associated with regional wall motion abnormality (WMA). Of note, repetitive doses of $0.25 \mathrm{mg}$ atropine and up to a dose of $1.0 \mathrm{mg}$ may be administered to achieve target heart rate; however, an intravenous beta-blocker agent must be ready to be administered to reverse the effects of dobutamine if needed [6].

Echocardiography is performed with harmonic imaging, and images acquired consist of the parasternal long and short axis views (PLAX and PSAX) and apical views, two-, three-, four- and five-chamber views (A2C, A3C, A4C and A5C). All acquired images may be displayed on a quad screen to allow side-by-side comparison at each stage $[4,5]$.

If baseline resting echocardiogram shows regional WMA, dobutamine is administered according to a low protocol in order to evaluate myocardial viability. With this protocol, initial dosage is $5 \mu \mathrm{g} / \mathrm{kg} / \mathrm{min}$, and then it is increased at $5-\mathrm{min}$ intervals to $10 \mu \mathrm{g} / \mathrm{kg} / \mathrm{min}$, then to $15 \mu \mathrm{g} / \mathrm{kg} / \mathrm{min}$ if required. The procedure may be switched to the standard protocol with a maximal dobutamine dose up to $40 \mu \mathrm{g} / \mathrm{kg} / \mathrm{min}$ according to clinical settings [8] (Fig. 1a, b).

\section{Steps of the procedure}

First, an intravenous line is placed, and electrodes are attached in order to get a real-time electrocardiography monitor, and 
Table 1. The Four Equation Possibilities in Response to Stress

\begin{tabular}{lll}
\hline Rest & Stress & Diagnosis \\
\hline Normokinesis & Normo- to hyperkinesis & Normal \\
Normokinesis & Hypokinesis to akinesis or dyskinesis & Ischemia \\
Akinesis & Hypo- to normokinesis & Viable \\
Akinesis or dyskinesis & Akinesis or dyskinesis & Necrosis \\
\hline
\end{tabular}

Table 2. Interpretation of DSE at Rest, With Low Dose Protocol and With Peak Dose

\begin{tabular}{llll}
\hline Nature of tissue & Rest & Low dose dobutamine & Peak dose dobutamine \\
\hline Normal segment & Normal & Normal or improves & Hyperkinetic \\
Ischemic segment & Normal & Normal or may worsen & Worsens \\
Viable ischemic & Hypo- or akinetic & Improves & Worsens (biphasic response) \\
Infarcted segment & Akinetic or dyskinetic & Akinetic or dyskinetic & Akinetic or dyskinetic \\
\hline
\end{tabular}

DSE: dobutamine stress echocardiography.

also a blood pressure automatic sphygmomanometer is placed. The room is made little dim in order to enhance image visibility. A resting electrocardiogram is performed, and then the patient is placed on his left side on an echo exam table in order to perform a resting echocardiogram. Afterward, dobutamine is administered while the physician continues to obtain echo images and cine loops to allow a post-test analysis. Sonographic images are taken at regular intervals (i.e. Q 3 - 5 min), while the personnel keeps monitoring symptoms, vital signs and electrocardiogram monitor [6].

Images are stored so that a post-exam analysis may be performed, and the whole test duration is about $1 \mathrm{~h}$. At the end of the test, intravenous line is removed once heart rate has returned to near normal value. After the test, there are no specific precautions that need to be taken except for warnings or symptoms that physician had made earlier.

In patients with suspected CAD, there are four responses expected with SEC according to wall motion response: normal, ischemic, viable and necrotic. The normal response consists of normokinetic segment at rest and that stays normal or becomes hyperkinetic during stress. A normal segment that worsens its function during stress reflects an ischemic response. A segment with resting dysfunction that remains fixed during stress reflects a necrotic response; of note, a resting akinesis which becomes dyskinesis during stress usually reflects a passive mechanical consequence of increased intraventricular pressure during SEC and should not be misinterpreted as a true active ischemia. In the viability response, a segment with resting akinesis may show either a sustained improvement during stress, indicating a non-jeopardized myocardium (stunned), or nonsustained improvement (early improvement with subsequent deterioration at peak, biphasic response), indicating a jeopardized region (hibernating myocardium) related to active viable segment that may improve after revascularization $[4,6]$. Table 1 summarizes the stress responses with the four equation possibilities. Table 2 shows the interpretation of DSE at rest, with low dose protocol and with peak dose dobutamine [4, 6] (Figs. 2 and 3 ).
The use of relatively new ultrasound technology helps in assessment of the different regional WMA, with more accurate quantification and localization of the WMA. Figure 4 shows

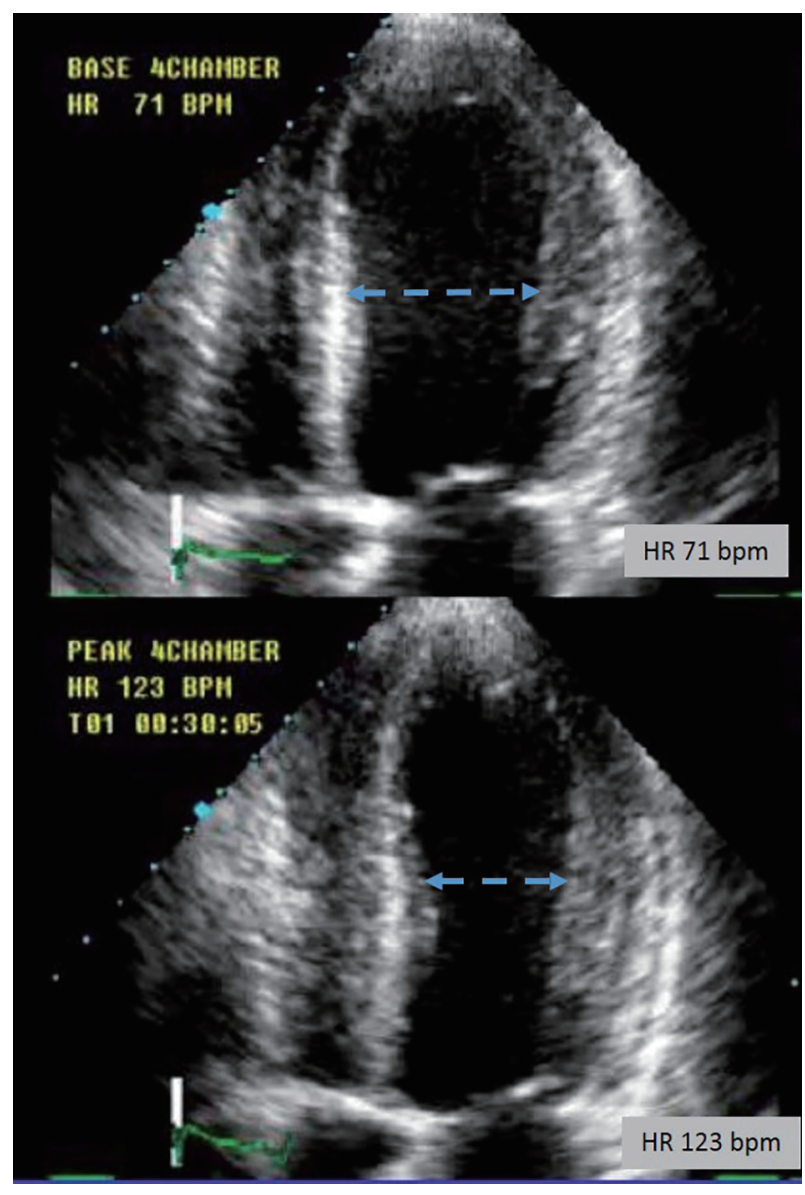

Figure 2. Apical four-chamber view showing the mid-left ventricular diameter change at rest and with peak DSE. DSE: dobutamine stress echocardiography. 

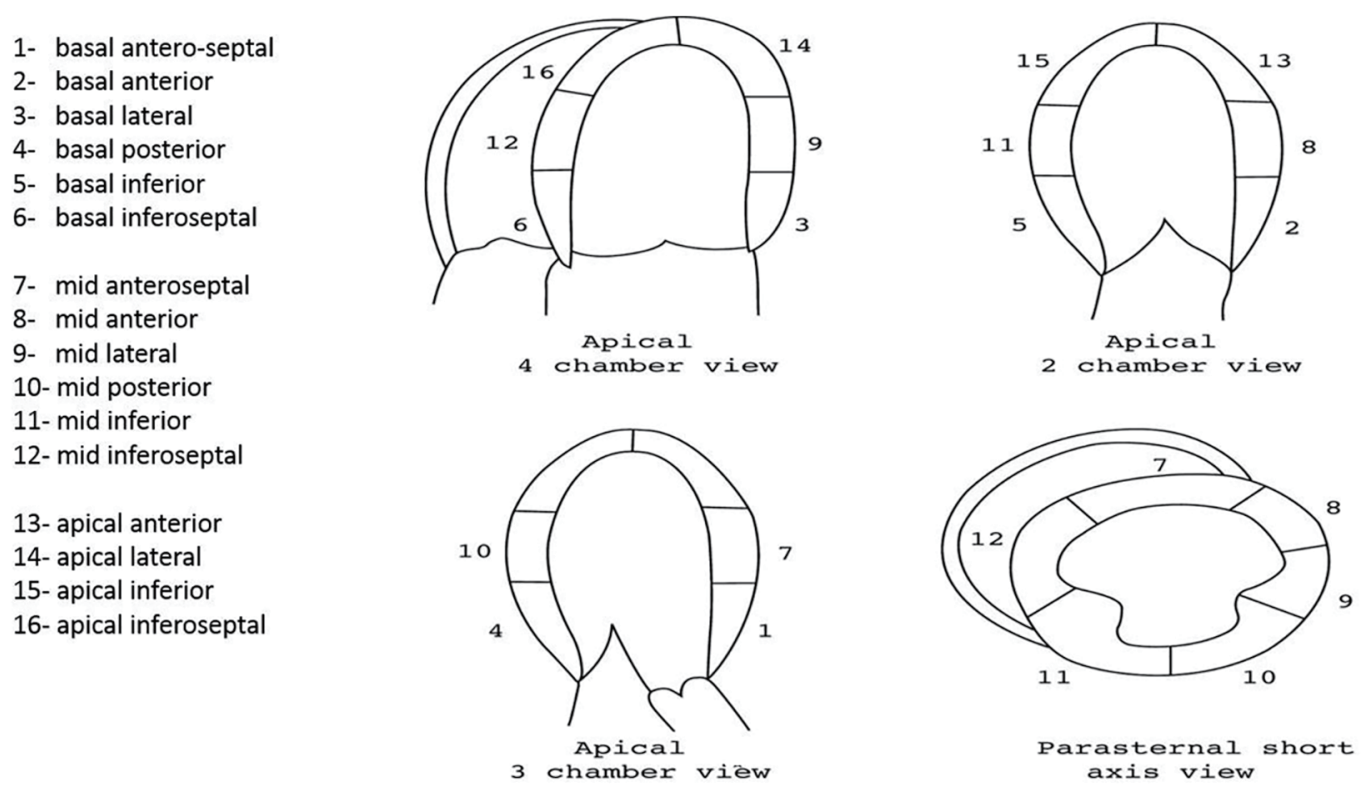

Figure 3. The different left ventricular myocardial segments explored during DSE. DSE: dobutamine stress echocardiography.

the benefit of automatic deformation imaging (DFI) analysis using the speckle tracking mode, with representation of the different segments in the Bull's eye figure (Fig. 5).

Use of DSE beyond CAD is well established as a technique and it has proven its benefit specifically in terms of assessment of valvular heart disease, allowing refining diagnosis, severity and prognosis, and also it helps in management planning based on changes in various parameters during DSE [9]. We hereby develop the use of SEC in patients with low flow low gradient aortic stenosis (LF/LG AS), the most common valvular heart disease that may require SEC [7,9]. Figure 6 shows the different potential causes of low flow across the aortic valve.

In patients with classical LF/LG AS, DSE is useful to distinguish patients with true severe AS from those with pseudosevere AS [10]. DSE in AS aims to increase cardiac output without inducing significant ischemia; accordingly, a very low

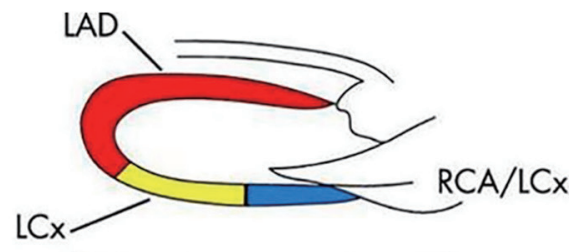

Long axis view

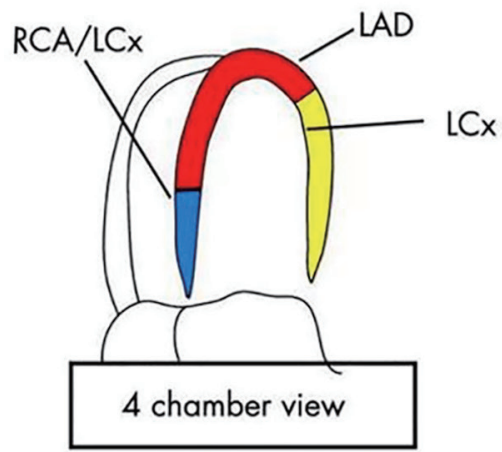

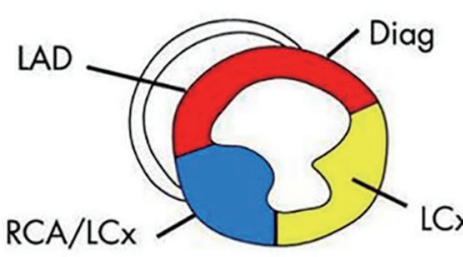
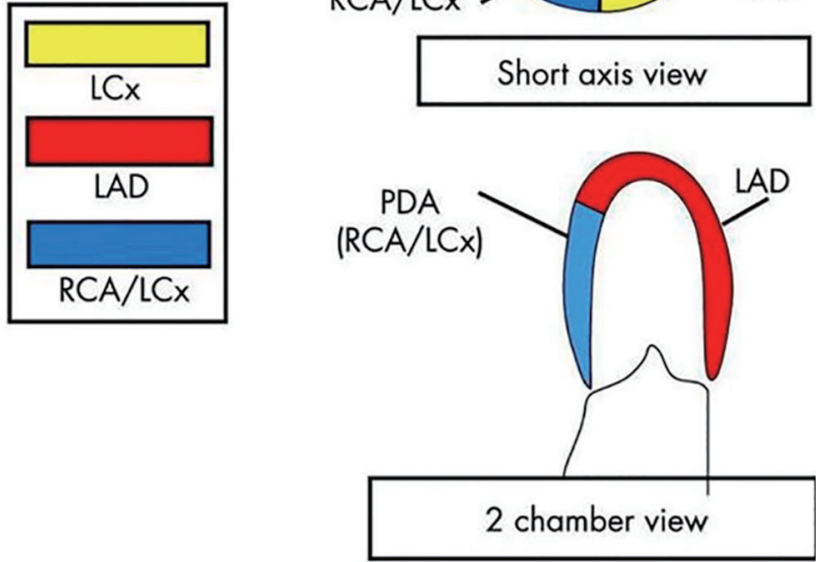

Figure 4. The different coronary territories with regard to left ventricular myocardial segments. LAD: left anterior descending; LCx: left circumflex; RCA: right coronary artery; Diag: diagonal branch. 


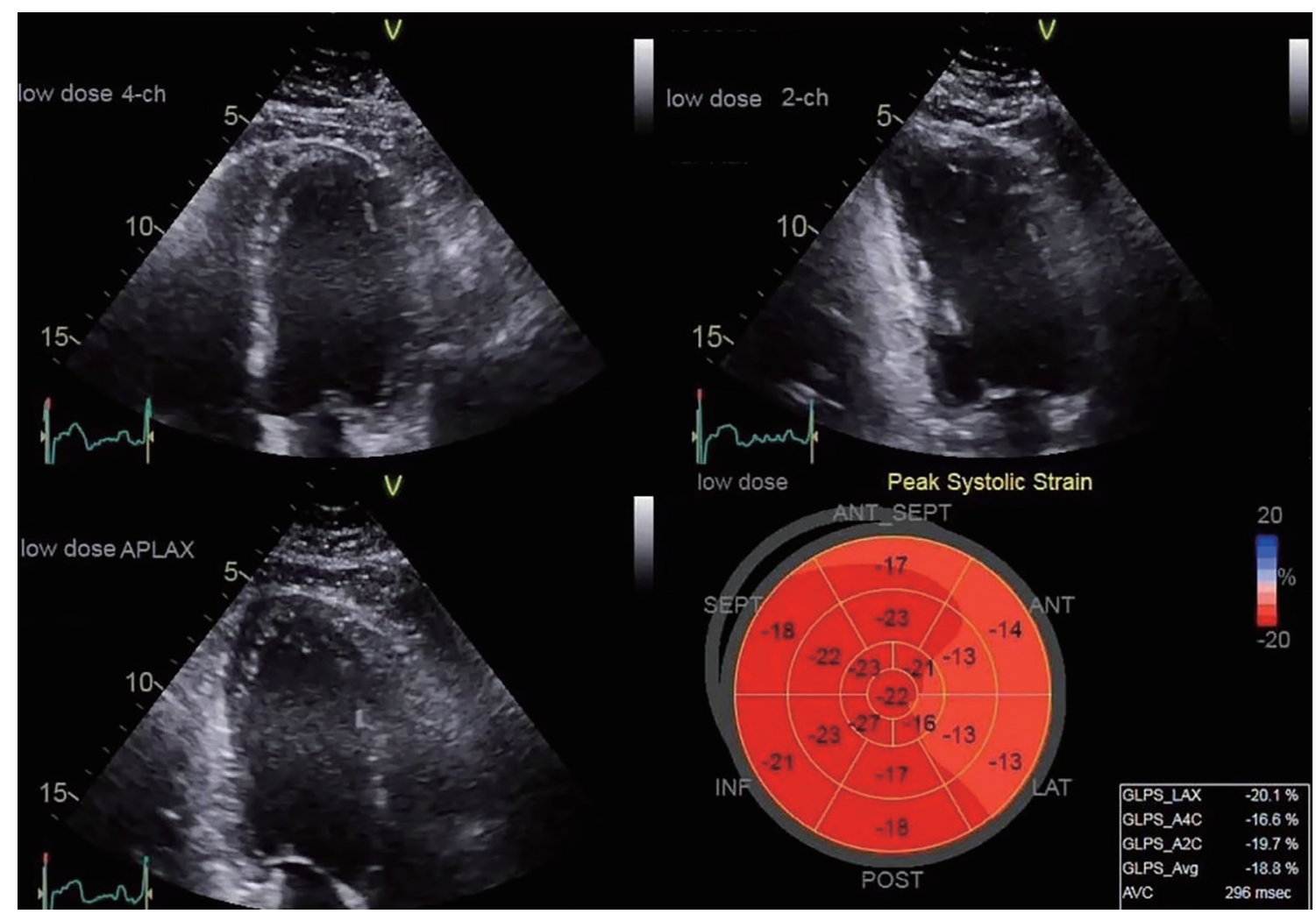

Figure 5. Deformation imaging method showing regional hypokinesis (mainly in the basal antero-septal, anterior and lateral segments) during a low dose DSE. DSE: dobutamine stress echocardiography.

dose dobutamine protocol is implemented, starting at a low dose $(2.5 \mu \mathrm{g} / \mathrm{kg} / \mathrm{min})$, and not exceeding $10 \mu \mathrm{g} / \mathrm{kg} / \mathrm{min}$. Infusion should be halted if pulse rises more than $20 \mathrm{bpm}$ over baseline or if it surpasses $100 \mathrm{bpm}$, and also the test is stopped when contractile reserve shows an increase of $20 \%$ or more. In all cases, the test must be stopped if the patient develops significant symptoms of test intolerance or impending signs of complications [4] (Fig. 7).

The classical views where data are acquired are $\mathrm{A} 3 \mathrm{C}$ or $\mathrm{A} 5 \mathrm{C}$ view, and stroke volume is better measured using the left ventricle outflow tract (LVOT) velocity time integral (VTI) and LVOT area. At every stage of the test, AV/VTI, LVOT/

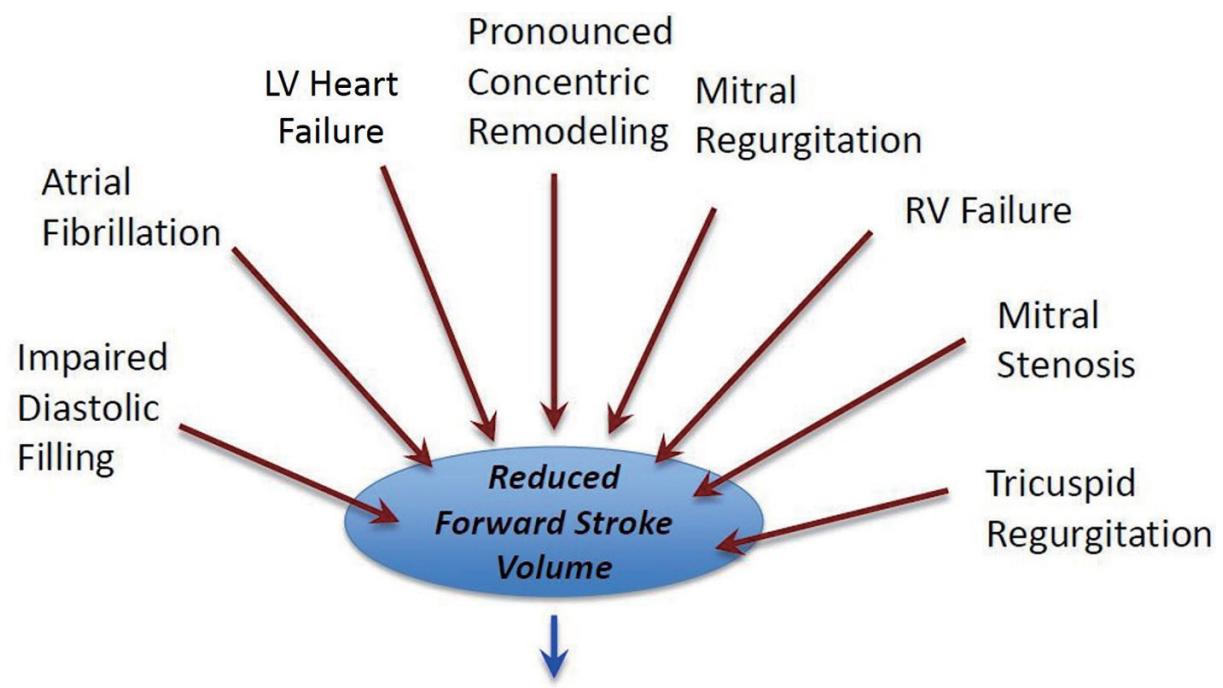

\section{Reduced Transvalvular flow rate}

Figure 6. The different causes that may result in transaortic low flow status. LV: left ventricle; RV: right ventricle. 


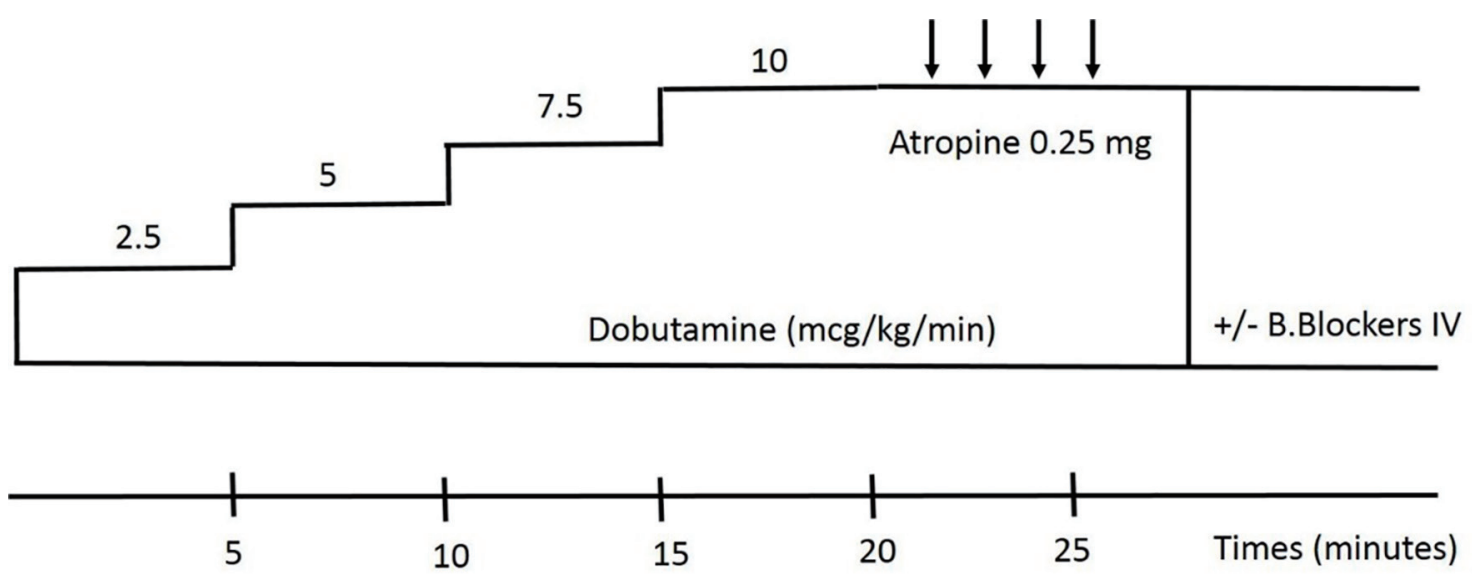

Figure 7. Very low dobutamine protocol.

Table 3. Changes in Echo Parameters During Stress

\begin{tabular}{lll}
\hline & True severe AS & Pseudo-severe AS \\
\hline Stroke volume and LVOT velocity & + & + \\
Transvalvular gradients & +++ & + \\
Aortic valve area & $=$ or $(-)$ & + \\
\hline
\end{tabular}

+: minor increase; +++: significant increase; =: similar; -: decrease. AS: aortic stenosis; LVOT: left ventricle outflow tract.

VTI, transvalvular mean and peak gradients, stroke volume and left ventricular ejection fraction (LVEF) are acquired and stored. The LVOT diameter is measured at baseline at mid-systole; continuity-equation is applied to measure the valve area at each stage during the test $[3,11]$. Adequate contractile reserve is considered present when LVEF or stroke volume rises by more than $20 \%$ during DSE; a noncompliant aortic valve may be moderately stenotic at rest but severely stenotic during stress given that cusps fail to open further.

During DSE, if the mean gradient rises to more than 40 $\mathrm{mm} \mathrm{Hg}$ while the aortic valve area remains $<1.0 \mathrm{~cm}^{2}$, DSE indicates true severe AS, whereas if the aortic valve area increases to more than $1.0 \mathrm{~cm}^{2}$, DSE implies pseudo-severe AS even if gradients may increase $[12,13]$ (Table 3 ). Similarly,

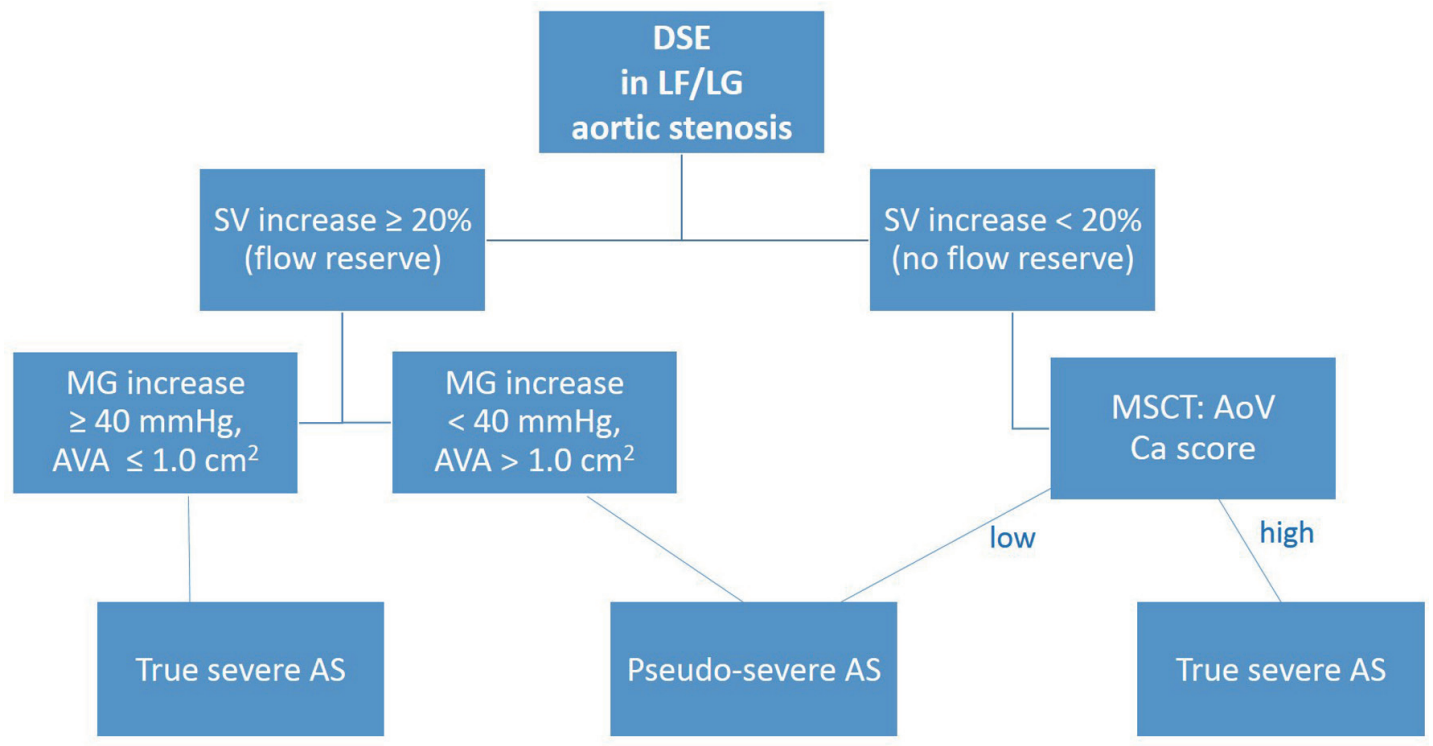

Figure 8. Algorithm showing the different alternatives and outcome while implementing DSE in LF/LG aortic stenosis. DSE: dobutamine stress echocardiography; SV: stroke volume; MSCT: multislice computed tomography; AoV: aortic valve; Ca: calcium; MG: mean gradient; AVA: aortic valve area. LF/LG: low flow low gradient. 
DSE may help to assess AS severity in patients with paradoxical LF/LG AS.

Aortic valve replacement should be considered in symptomatic patients with $\mathrm{LF} / \mathrm{LG}$ with reduced $\mathrm{LVEF}$ and with evidence of contractile (or flow) reserve. However, if there is no significant contractile reserve, it will be difficult or even impossible to make the difference between true-severe and pseudo-severe AS [11]. Figure 8 shows the algorithm of assessment of LF/LG AS.

\section{Obstacles and Outcome}

\section{Caution, side effects and contraindications (obstacles)}

SEC is an interventional, though noninvasive procedure, during which heart rate is increased using either pharmacological intervention. Accordingly, the test may be associated with side effects related either to the drug and/or to the underlying cardiac conditions (i.e. stress arrhythmia, angina). In this respect, it is essential to conform with international standards regarding the indications and precautions of the test [11]. DSE should be used with caution and could be potentially contraindicated if the LV has severe heart failure or severe LV hypertrophy; in this latter case, there is a risk of dobutamineinduced LVOT obstruction and hemodynamic shock during the test [14].

DSE should always be performed in presence of a cardiologist with expertise in the field. Non-exercise stress testing has nearly an equivalent safety as exercise testing, with a risk of serious complications being negligible in experienced centers. Patient should be in a fasting status for at least $6 \mathrm{~h}$ before the test. Previous adverse reaction to the drug planned to be administered is a precaution or even a contraindication to the test; recent changes in cardiac symptoms, such as the recent onset of chest pain or dyspnea may imply unstable coronary syndrome and constitute a contraindication to the test. Patients with chronotropic incompetence may not have an adequate chronotropic response to DSE and therefore the test may be non-interpretable. Patients with pacemakers may have inadequate rate response, and also these patients may exhibit regional wall abnormalities owing to pacing effect, and therefore the test may be difficult to analyze [6].

Patients usually may continue their regular medications, except for those required to be halted for better test results, such as amiodarone, calcium channel blockers, beta-blockers or nitrates which are halted at least $24 \mathrm{~h}$ before the test. Moreover, medications containing caffeine may influence test results and therefore should be avoided. Similarly, nicotine may interfere with the test results and smoking is better avoided $24 \mathrm{~h}$ before the test [6].

Most side effects are minor but could be limiting to continue the test, as side effects may preclude the achievement of maximal pharmacological stress in nearly $10 \%$ of patients with DSE [6]. Most commonly encountered side effects with DSE are warm feeling, flush, palpitations related to tachycardia, and in some cases, a mild headache. In rare instances, patients may have a residual sensitivity to bright light for an hour or so fol- lowing test. Of note, and whatsoever are the facility settings, an informed consent should be signed before the test.

Sonographic noise and time-consuming analysis are issues that still need to be addressed when using advanced echo techniques such as speckle tracking and 3D echocardiography; moreover, 3D echo derived data have currently insufficient temporal resolution for the high heart rate during DSE. Alternatives to SEC include stress scintigraphy, stress cardiac perfusion magnetic resonance imaging and stress single-photon emission computed tomography (SPECT) [5].

\section{Outcome}

\section{Diagnostic accuracy and prognostic value}

Initial assessment for diagnosis of ischemia with DSE is based on eyeballing interpretation of regional WMA. However, more objective and quantitative approach is needed to diagnose and quantify regional myocardial function [15]. In terms of CAD, the main response regarding regional WMA is one of four: normal, ischemic, viable and necrotic; usually, WMA in at least two adjacent segments is required to confirm test positivity.

Tissue Doppler imaging (TDI) and DFI are newer techniques available for this purpose and have demonstrated a high sensitivity to detect regional ischemia and to predict patient outcome [16]. Of note, very high heart rates often preclude accurate analysis of WMA owing to low temporal resolution, and combination of TDI and DFI helps to better identify WMA and predict myocardial viability, with a high accuracy when using the low dose dobutamine protocol [8].

Consistency in interpreting DSE is essential and low volume echocardiography laboratories are the most vulnerable in this regard. Heterogeneity in data acquisition and assessment criteria may lead to low agreement in interpretation of DSE and therefore, standardization of image acquisition and reading criteria of DSE is recommended [17]. Importantly, a prospective study addressing SEC is currently lacking; Picano et al are conducting an ongoing prospective SEC study aiming to investigate technical questions such as feasibility, safety and reproducibility in various clinical scenarios [18].

\section{Conclusion}

SEC is a useful tool that yields substantial diagnostic and prognostic information in patients with $\mathrm{CAD}$, derived from regional wall motion responses to stress. In addition, SEC provides valuable diagnostic and prognostic information in many cardiovascular conditions such as microvascular CAD, valvular heart diseases and diastolic dysfunction.

SEC is technically challenging and its use should be restricted to centers with high volume, and also its use by occasional practitioners rather than experts may be accompanied with loss of accuracy. In this regard, the development of this technique depends on better medical training, along with technical developments in order to address all situations with professionalism, safety and accuracy. 


\section{Acknowledgments}

None to declare.

\section{Financial Disclosure}

None to declare.

\section{Conflict of Interest}

None to declare.

\section{Author Contributions}

AK designed the research, made data collection and wrote the first draft. AK, EB and MK analyzed the data, created figures and tables. EB and MK made relevant bibliographical search. AK provided expert clinical knowledge and opinion. All authors revised and approved the final submitted version.

\section{Data Availability}

The authors declare that data supporting the findings of this study are available within the article.

\section{Abbreviations}

SEC: stress echocardiography; CAD: coronary artery disease; DSE: dobutamine stress echocardiography; WMA: wall motion abnormality; LF/LG AS: low flow low gradient aortic stenosis; LVOT: left ventricle outflow tract; VTI: velocity time integral; LVEF: left ventricular ejection fraction; TDI: tissue Doppler imaging; DFI: deformation imaging

\section{References}

1. Armstrong WF, Ryan T. Stress echocardiography from 1979 to present. J Am Soc Echocardiogr. 2008;21(1):2228.

2. Armstrong WF, Zoghbi WA. Stress echocardiography: current methodology and clinical applications. J Am Coll Cardiol. 2005;45(11):1739-1747.

3. Picano E, Pellikka PA. Stress echo applications beyond coronary artery disease. Eur Heart J. 2014;35(16):10331040.

4. Sicari R, Nihoyannopoulos P, Evangelista A, Kasprzak J, Lancellotti P, Poldermans D, Voigt JU, et al. Stress echocardiography expert consensus statement - executive summary: European Association of Echocardiography (EAE) (a registered branch of the ESC). Eur Heart J. 2009;30(3):278-289.
5. Becher H, Chambers J, Fox K, Jones R, Leech GJ, Masani $\mathrm{N}$, Monaghan M, et al. BSE procedure guidelines for the clinical application of stress echocardiography, recommendations for performance and interpretation of stress echocardiography: a report of the British Society of Echocardiography Policy Committee. Heart. 2004;90(Suppl 6):vi23-30.

6. Marwick TH. Stress echocardiography. Heart. 2003;89(1):113-118.

7. Lancellotti P, Dulgheru R, Go YY, Sugimoto T, Marchetta S, Oury C, Garbi M. Stress echocardiography in patients with native valvular heart disease. Heart. 2018;104(10):807-813.

8. Bansal M, Jeffriess L, Leano R, Mundy J, Marwick TH. Assessment of myocardial viability at dobutamine echocardiography by deformation analysis using tissue velocity and speckle-tracking. JACC Cardiovasc Imaging. 2010;3(2):121-131.

9. Pierard LA, Lancellotti P. Stress testing in valve disease. Heart. 2007;93(6):766-772.

10. Czarny MJ, Resar JR. Diagnosis and management of valvular aortic stenosis. Clin Med Insights Cardiol. 2014;8(Suppl 1):15-24.

11. Yavagal ST, Deshpande N, Admane P. Stress echo for evaluation of valvular heart disease. Indian Heart J. 2014;66(1):131-138.

12. Petkow Dimitrow P, Cotrim C, Cheng TO. Need for a standardized protocol for stress echocardiography in provoking subaortic and valvular gradient in various cardiac conditions. Cardiovasc Ultrasound. 2014;12:26.

13. Dimitrova K, Kossaify A. Echocardiogram for aortic valve stenosis, the complex interplay between flow and gradient and insight into stress echocardiography and transoesophageal echo for Transcatheter Aortic Valve Implantation (TAVI). Sch J App Med Sci. 2016;4(7B):24232427.

14. Garbi M, Chambers J, Vannan MA, Lancellotti P. Valve stress echocardiography: a practical guide for referral, procedure, reporting, and clinical implementation of results from the HAVEC group. JACC Cardiovasc Imaging. 2015;8(6):724-736.

15. Mada RO, Duchenne J, Voigt JU. Tissue Doppler, strain and strain rate in ischemic heart disease "how I do it". Cardiovasc Ultrasound. 2014;12:38.

16. Bjork Ingul C, Stoylen A, Slordahl SA, Wiseth R, Burgess M, Marwick TH. Automated analysis of myocardial deformation at dobutamine stress echocardiography: an angiographic validation. J Am Coll Cardiol. 2007;49(15):1651-1659.

17. Hoffmann R, Lethen H, Marwick T, Arnese M, Fioretti P, Pingitore A, Picano E, et al. Analysis of interinstitutional observer agreement in interpretation of dobutamine stress echocardiograms. J Am Coll Cardiol. 1996;27(2):330336.

18. Picano E, Ciampi Q, Citro R, D'Andrea A, Scali MC, Cortigiani L, Olivotto I, et al. Stress echo 2020: the international stress echo study in ischemic and non-ischemic heart disease. Cardiovasc Ultrasound. 2017;15(1):3. 